
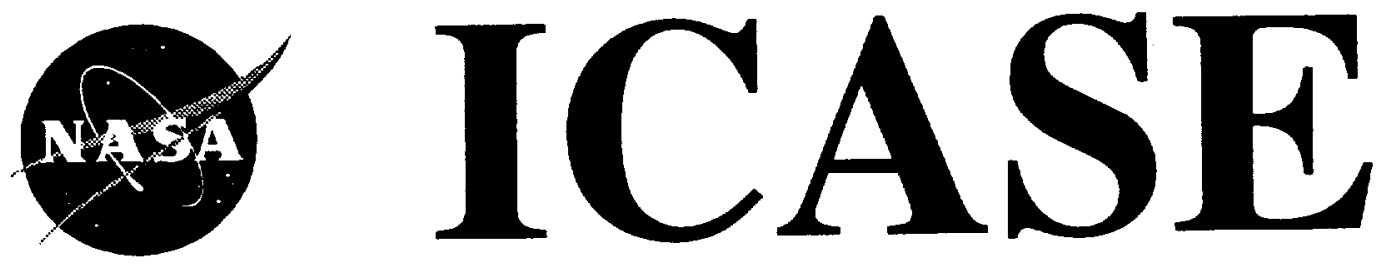

\title{
PRESSURE UPDATING METHODS FOR THE STEADY-STATE FLUID EQUATIONS
}

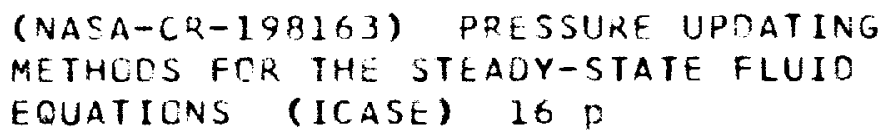

$N 95-30353$

Unclas

\section{A. Fiterman \\ E. Turkel \\ V. Vatsa}

Contract No. NAS1-19480

May 1995

Institute for Computer Applications in Science and Engineering NASA Langley Research Center Hampton, VA 23681-0001

SRA Operated by Universities Space Research Association 



\title{
PRESSURE UPDATING METHODS FOR THE STEADY-STATE FLUID EQUATIONS
}

\author{
A. Fiterman \\ Department of Environmental Sciences and Energy Research \\ Weizmann Institute, Rechovot, Israel \\ E. Turkel* \\ Institute for Computer Applications in Science and Engineering and \\ School of Mathematical Science, Tel-Aviv University \\ Ramat-Aviv, Tel-Aviv, Israel \\ V. Vatsa \\ NASA Langley Research Center \\ Hampton, VA
}

\begin{abstract}
We consider the steady state equations for a compressible fluid. Since we wish to solve for a range of speeds we must consider the equations in conservation form. For transonic speeds these equations are of mixed type. Hence, the usual approach is to add time derivatives to the steady state equations and then march these equations in time. One then adds a time derivative of the density to the continuity equation, a derivative of the momentum to the momentum equation and a derivative of the total energy to the energy equation. This choice is dictated by the time consistent equations. However, since we are only interested in the steady state this is not necessary. Thus we shall consider the possibilty of adding a time derivative of the pressure to the continuity equation and similar modifications for the energy equation. This can then be generalized to adding combinations of time derivatives to each equation since these vanish in the steady state. When using acceleration techniques such as residual smoothing, multigrid, etc. these are applied to the pressure rather than the density. Hence, the code duplicates the behavior of the incompressible equations for low speeds.
\end{abstract}

\footnotetext{
*This research was supported by the National Aeronautics and Space Administration under NASA Contract No. NAS119480 while the second author was in residence at the Institute for Computer Applications in Science and Engineering (ICASE), NASA Langley Research Center, Hampton, VA 23681-0001.
} 



\section{Introduction}

It is well known, that it is difficult to solve the compressible equations for low Mach numbers. For an explicit scheme this is easily seen by inspecting the time steps. For stability, the time step must be chosen inversely proportional to the largest eigenvalue of the system which, for slow flows, is approximately the speed of sound, c. However, other waves are convected at the fluid speed, $u$, which is much slower. Hence, these waves don't change very much over a time step. Thousands of time steps may be required to reach a steady state. Should one try a multigrid acceleration one finds that the same disparity in wave speeds slows down the multigrid acceleration. With an implicit method an ADI factorization is generally used so that one can easily invert the implicit factors. The use of ADI introduces factorization errors which again slows down the convergence rate when there are wave speeds of very different magnitudes [7] .

We consider systems of the form

$$
w_{t}+f_{x}+g_{y}=0 \text {. }
$$

Our analysis will be based on the linearized equations so that the conservation form does not appear in the analysis though it does appear in the final numerical approximation. This system is now replaced by

$$
\mathbf{P}^{-1} w_{t}+f_{x}+g_{y}=0
$$

or in linearized form

$$
\mathbf{P}^{-1} w_{t}+A w_{x}+B w_{y}=0,
$$

with $\mathrm{A}$ and $\mathrm{B}$ constant matrices.

In order for this system to be equivalent to the original system, in the steady state, we demand that $\mathbf{P}^{-1}$ have an inverse. This only need be true in the flow regime under consideration. We shall see later that frequently $\mathbf{P}$ is singular at stagnation points and also along sonic lines. Thus, the final preconditioner will be smoothed out in the vicinity of points where $M=0$ or $M=1$.

Assuming the steady state has a unique solution, it does not matter which system we march to a steady state. We shall later see that for the finite difference approximations the steady state solutions are not necessarily the same and usually the preconditioned system leads to a better behaved steady state.

\section{The Incompressible Limit}

The time dependent two dimensional Euler equations can be written as

$$
\begin{aligned}
p_{t}+u p_{x}+v p_{y}+\rho a^{2}\left(u_{x}+v_{y}\right) & =0 \\
u_{t}+u u_{x}+v u_{y}+\frac{p_{x}}{\rho} & =0 \\
v_{t}+u v_{x}+v v_{y}+\frac{p_{y}}{\rho} & =0 \\
S_{t}+u S_{x}+v S_{y} & =0
\end{aligned}
$$

The form of this system is unchanged if we nondimensionalize the equations. From now on we shall assume that $u, v, p, \rho$ are nondimensional quantities where the dimensional variables are nondimensionalized by $u_{*}, p_{*}, \rho_{*}$. Following [4] we define $\epsilon=\frac{u_{*}}{a_{*}}=M_{*}$. If the fluid is isentropic then

$$
p=\frac{\rho^{\gamma}}{\gamma \epsilon^{2}} \quad, \quad a=\frac{\rho^{\frac{\gamma-1}{2}}}{\epsilon}
$$

Hence, as $\epsilon$ goes to zero the speed of sound, a, goes to infinity and so the first equation in (2) reduces to $u_{x}+v_{y}=0$. 
It was pointed out in ([10], [11]) that these equations can be symmetrized by using $\frac{d p}{p a}$ as the independent variable rather than $d p$. Hence, we define $\phi$ by $d \phi=\frac{d p}{\rho a}$. For isentropic flow both $\mathrm{p}$ and a are functions only of the density and so using (3) this can be integrated explicitly. This gives $\phi=\frac{\frac{\rho-1}{2}}{\frac{\gamma-1}{2} \epsilon}$. As the Mach number goes to zero $\phi$ tends to infinity and therefore, Gustafsson and Stoor [4] subtract a constant and define

$$
\phi=\frac{\rho^{\frac{\gamma-1}{2}}-1}{\frac{\gamma-1}{2} \epsilon}
$$

This amounts to specifying the constant in the integration of $d \phi$ from $d p$. They then prove, using energy methods, that

$$
a \phi_{x} \rightarrow \frac{\partial p_{\text {incompressible }}}{\partial x}
$$

Hence, $\phi$ and all its derivatives behave as $O(M)$ as $M \rightarrow 0$. Since $\rho \rightarrow 1$ and using the definition of $d \phi$ this is equivalent to

$$
d p_{\text {compressible }} \rightarrow d p_{\text {incompressible }}
$$

We now consider how to construct a matrix artificial viscosity that will enable us to reach the incompressible limit. Consider

$$
\mathbf{P}^{-1} w_{t}+f_{x}+g_{y}=h\left[\left(Q_{1} w_{x}\right)_{x}+\left(Q_{2} w_{y}\right)_{y}\right]
$$

We wish to find the dependence of $\mathbf{P}$ and $Q_{i}$ on the Mach number as $M \rightarrow 0$ so that we get the proper convergence. We therefore consider the isentropic equations based on $w=(\phi, u, v)$ see (4). This has the symmetric form

$$
\begin{aligned}
& \mathbf{P}^{-1} w_{t}+\left(\begin{array}{lll}
a_{11} & a_{12} & a_{13} \\
a_{12} & a_{22} & a_{23} \\
a_{11} & a_{23} & a_{33}
\end{array}\right) w_{x} \\
& +\left(\begin{array}{lll}
b_{11} & b_{12} & b_{13} \\
b_{12} & b_{22} & b_{23} \\
b_{11} & b_{23} & b_{33}
\end{array}\right) w_{y}=h\left[\left(Q_{1} w_{x}\right)_{x}+\left(Q_{2} w_{y}\right)_{y}\right]
\end{aligned}
$$

As $M \rightarrow 0, a_{12}$ and $b_{13}=O(1 / M)$ while $d \phi=O(M)$ while all other quantities are bounded. Hence, the leading terms in the first equation are all $O(1 / M)$ while they are $O(1)$ for the second and third equations. Multiplying the first equation by $M$ and taking the limit we get $u_{x}+v_{y}$ for the space derivatives on the left hand side. Using $d \phi=O(M), d u=O(1), d v=O(1)$ we see that a necessary condition for convergence as $M \rightarrow 0$ is that $P^{-1}, Q_{i}$ have the form

$$
\mathbf{P}^{-1}, \mathbf{Q}_{1}, \mathbf{Q}_{2} \sim\left(\begin{array}{ccc}
O\left(\frac{1}{M^{2}}\right) & O\left(\frac{1}{M}\right) & O\left(\frac{1}{M}\right) \\
O\left(\frac{1}{M}\right) & O(1) & O(1) \\
O\left(\frac{1}{M}\right) & O(1) & O(1)
\end{array}\right)
$$

The artificial viscosity matrices $Q_{i}$ are related to the preconditioner. Consider the one dimensional equation

$$
u_{t}+P f_{x} \sim\left(|Q| u_{x}\right)_{x}
$$

Let $A=\frac{\partial f}{\partial u}$. Since we are updating $P f$ we should have $Q=P A$. However, this is not in conservation form at the steady state. Instead we consider artificial viscosities of the form

$$
P^{-1} u_{t}+f_{x} \sim\left(P^{-1}\left(|P A| u_{x}\right)_{x}\right.
$$

or

$$
u_{t}+P f_{x} \sim P\left(P^{-1}\left(|P A| u_{x}\right)_{x}\right.
$$


This would be equivalent to the original form if $\mathbf{P}$ were constant. Instead we have terms like $\mathbf{P}_{\mathbf{i}} \mathbf{P}^{-\mathbf{1}} \mathbf{i + 1 / 2}$ that appear.

We note that the conditions on the matrix $\left(\mathbf{P}^{-1}|\mathbf{P D}|\right)$ comp are not satisfied by the non-preconditioned Roe matrices. Furthermore, even reasonable preconditioners need not satisfy these conditions. Consider, for example, the one dimensional system

$$
\mathbf{P}^{-1} w_{t}+A w_{x}=h\left(Q w_{x}\right)_{x}
$$

A reasonable choice is $\mathbf{P}^{-1}=|A|$ i.e. $\mathbf{P}=\left|A^{-1}\right|$. In this case all the wave speeds of $\mathbf{P} A$ are \pm 1 . Now

$$
\begin{aligned}
& Q=\mathbf{P}^{-1}|\mathbf{P} A|=\left.|A||| A\right|^{-1} A|=| A \mid \sim \\
& \left(\begin{array}{ccc}
O\left(\frac{1}{M}\right) & O(1) & O(1) \\
O(1) & O\left(\frac{1}{M}\right) & O(1) \\
O(1) & O(1) & O(1)
\end{array}\right),
\end{aligned}
$$

i.e. $Q$ is the nonpreconditioned Roe matrix which does not have the desired property. We therefore conclude that for an upwind difference scheme the Riemann solver should be based on the preconditioned system and not the original scheme. In [3] plots are shown to illustrate the greatly improved accuracy for low Mach number flows when the Riemann solver is based on the preconditioning. Characteristics in the boundary conditions these should be based on the characteristics of the modified system and not the physical system. Preconditioning is even more important when using multigrid than with an explicit scheme. With the original system the disparity of the eigenvalues greatly affects the smoothing rates of the slow components and so slows down the multigrid method, [6].

We conclude from the above remarks that the steady state solution of the preconditioned system may be different from that of the physical system. Thus, on the finite difference level the preconditioning can improve the accuracy as well as the convergence rate.

\section{Algorithm}

In terms of the primitive variables the preconditioning we consider is:

$$
\begin{aligned}
& \left(\begin{array}{cccc}
\frac{a^{2}}{\beta^{2}} & 0 & 0 & 0 \\
\frac{\alpha a u}{\beta^{2}} & 1 & 0 & 0 \\
\frac{\alpha a v}{\beta^{2}} & 0 & 1 & 0 \\
0 & 0 & 0 & 1
\end{array}\right)\left(\begin{array}{c}
\frac{d p}{\rho a} \\
d u \\
d v \\
d S
\end{array}\right)_{t} \\
& +\left(\begin{array}{llll}
u & a & 0 & 0 \\
a & u & 0 & 0 \\
0 & 0 & u & 0 \\
0 & 0 & 0 & u
\end{array}\right)\left(\begin{array}{c}
\frac{d p}{\rho a} \\
d u \\
d v \\
d S
\end{array}\right)_{x} \\
& +\left(\begin{array}{llll}
v & 0 & a & 0 \\
0 & v & 0 & 0 \\
a & 0 & v & 0 \\
0 & 0 & 0 & v
\end{array}\right)\left(\begin{array}{l}
\frac{d p}{\rho a} \\
d u \\
d v \\
d S
\end{array}\right)_{y}=0
\end{aligned}
$$

The nonpreconditioned case corresponds to $\beta^{2}=a^{2}, \alpha=0$. Let $q=u \omega_{1}+v \omega_{2}$, then the eigenvalues of PD are given by

$$
\begin{aligned}
& d_{0}=q \quad \text { (double) } \\
& d_{ \pm}=\frac{q}{2}\left[\left(1-\alpha+\beta^{2} / a^{2}\right) \pm\right. \\
& \left.\sqrt{\left(1-\alpha+\beta^{2} / a^{2}\right)^{2}+4\left(\omega_{1}^{2}+\omega_{2}^{2}-q^{2} / a^{2}\right) \frac{\beta^{2}}{q^{2}}}\right]
\end{aligned}
$$


For general curvilinear coordinates, in the "i" direction $\omega_{1}=y_{\eta}, \omega_{2}=-x_{\eta}$. The time step is bounded by $\frac{V O L}{d_{+}}$.

Our ultimate goal is to have a compressible code that solves the incompressible equations when the input Mach number is zero. So we wish to use variables that give us the same result as an incompressible code on all levels of the algorithm, e.g. flux computation, boundary conditions, acceleration techniques, etc. Hence, we choose as our basic variables

$$
\begin{aligned}
& W_{p}=\left(\begin{array}{c}
p^{\prime} \\
\rho u \\
\rho v \\
E^{\prime}
\end{array}\right), \quad Q=\left(\begin{array}{c}
p^{\prime} \\
\rho u \\
\rho v \\
H^{\prime}
\end{array}\right) \\
& F=\left(\begin{array}{c}
\rho u \\
\rho u^{2}+p^{\prime} \\
\rho u v \\
\rho H^{\prime} u
\end{array}\right), \quad G=\left(\begin{array}{c}
\rho v \\
\rho u v \\
\rho v^{2}+p^{\prime} \\
\rho H^{\prime} v
\end{array}\right), \\
& \frac{\partial W_{p}}{\partial t}=\mathbf{P} \mathbf{p}\left[\frac{\partial F}{\partial x}+\frac{\partial G}{\partial y}\right] .
\end{aligned}
$$

where

$$
\begin{aligned}
p^{\prime}= & p-p_{\infty} \quad h_{\infty}=\frac{a^{2}}{\gamma-1} \\
E^{\prime}= & c_{p} \rho\left(T-T_{\infty}\right)-\left(p-p_{\infty}\right)+\frac{\rho\left(u^{2}+v^{2}\right)}{2} \\
& =E+p_{\infty}-h_{\infty} \rho \\
\rho H^{\prime}= & E^{\prime}+p^{\prime}=E+p-h_{\infty} \rho
\end{aligned}
$$

We subtract the constants to keep the quantities in scale. Density is now calculated from the pressure and total energy. Because the modified energy $E^{\prime}$ also contains the density we get a quadratic equation for the density. Choosing the positive square root guarantees that the density is always positive. The residual smoothing and multigrid are applied to $p^{\prime}$ and $E^{\prime}$ rather than $\rho$ and $E$. Thus, we duplicate the treatment of the variables in a pseudo-compressible incompressible code.

$$
\mathbf{P}_{p}=\mathbf{I}+\Delta \cdot\left(\begin{array}{cccc}
1-\frac{1}{\Delta} & \frac{-u}{G+h_{\infty}} & \frac{-v}{G+h_{\infty}} & \frac{1}{G+h_{\infty}} \\
-u B_{2} & \frac{u^{2} B_{2}}{G+h_{\infty}} & \frac{u v B_{2}}{G+h_{\infty}} & \frac{-u B_{2}}{G+h_{\infty}} \\
-v B_{2} & \frac{u v B_{2}}{G+h_{\infty}} & \frac{v B_{2}}{G+h_{\infty}} & \frac{-v B_{2}}{G+h_{\infty}} \\
-B_{4} & \frac{u B}{G+h_{\infty}} & \frac{v B_{1}}{G+h_{\infty}} & \frac{-B_{1}}{G+h_{\infty}}
\end{array}\right)
$$

where $h=c_{p} T=\frac{a^{2}}{\gamma-1}, G=\frac{u^{2}+v^{2}}{2}, \Delta=\frac{\left(G+h_{\infty}\right) \beta^{2}}{h}$, In the appendix we derive this form of $\mathbf{P}_{p}$.

$$
\begin{aligned}
& B_{1}=\frac{1}{\beta^{2}}-\frac{1}{(\gamma-1) h}=\frac{1}{\beta^{2}}-\frac{1}{a^{2}} \\
& B_{2}=B_{1}+\frac{\alpha}{\beta^{2}} \\
& B_{4}=B_{1} H^{\prime}+\frac{\alpha\left(u^{2}+v^{2}\right)}{\beta^{2}}
\end{aligned}
$$


We choose $\beta^{2}=\min \left\{\max \left[\beta_{1}\left(u^{2}+v^{2}\right), \beta_{\min }^{2}\right], a^{2}\right\}$ where $\beta_{\min }$ should have the units of speed. The choice of $\beta_{\min }$ is discussed in the result section. In all cases $\alpha=\min \left[1, \frac{\beta^{2}}{\beta_{1}\left(u^{2}+v^{2}\right)}\right]$. We can evaluate this efficiently by defining $S=\Delta \cdot\left(d \rho-\frac{u d(\rho u)+v d(\rho v)-d E}{G+h_{\infty}}\right)=\frac{\beta^{2} d p}{(\gamma-1) h}$. Then

$$
\begin{aligned}
d p_{\text {new }}^{\prime} & =S \\
d(\rho u)_{\text {new }} & =d(\rho u)_{\text {orig }}-B_{2} u S \\
d(\rho v)_{\text {new }} & =d(\rho v)_{\text {orig }}-B_{2} v S \\
d E_{\text {new }}^{\prime} & =d E_{\text {orig }}-B_{4} S
\end{aligned}
$$

These equations are given for the nondimensionalized variables. The nondimensionalization affects the convergence. In some codes, $\mathrm{p}$ and $\rho$ are fixed in the far field. This implies that the speed of sound, a, is also bounded. As the Mach number goes to zero the pressure remains of order 1 while the velocities go to zero. Alternatively, one can nondimensionalize so that the velocities are of order 1 in the far field and then the pressure and speed of sound go to infinity, unless one subtracts an appropriate constant,

The boundary conditions at the far field boundary, for subsonic flow, are based on the one dimensional theory of characteristics in the direction normal to the boundary. The preconditioning changes the form of these characteristic variables. In differential form they are given by

$$
\begin{array}{r}
R_{1}=d u-\frac{1}{2 \rho \beta^{2}}\left(u\left(1-\alpha-\frac{\beta^{2}}{c^{2}}\right)\right. \\
\left.-\sqrt{\left.\left(u\left(1-\alpha+\frac{\beta^{2}}{c^{2}}\right)\right)^{2}+4\left(1-\frac{u^{2}}{c^{2}}\right) \beta^{2}\right)}\right) d p^{\prime} \\
R_{2}=d u-\frac{1}{2 \rho \beta^{2}}\left(u\left(1-\alpha-\frac{\beta^{2}}{c^{2}}\right)\right. \\
\left.+\sqrt{\left(u\left(1-\alpha+\frac{\beta^{2}}{c^{2}}\right)\right)^{2}+4\left(1-\frac{u^{2}}{c^{2}}\right) \beta^{2}}\right) d p^{\prime}
\end{array}
$$

where $\mathrm{u}$ is the component of the velocity normal to the boundary. If we consider low Mach numbers then we can approximate these by

$$
R_{1}=d u+\frac{d p^{\prime}}{\rho \beta}, \quad R_{2}=d u-\frac{d p^{\prime}}{\rho \beta}
$$

which is the same as for the incompressible case. Hence, at inflow $R_{1}, v$ (tangential velocity) and $S$ are specified while $R_{2}$ is extrapolated from the interior. We then calculate u (normal velocity) and the pressure from $R_{1}$ and $R_{2}$ and then the density and total energy. At outflow the role of specified and extrapolated quantities is reversed. At solid boundaries the normal momentum equation is used which is not affected by the preconditioning.

\section{Computational Results}

The solution is advanced by a explicit Runge-Kutta method ([5],[8]) with residual smoothing and multigrid and no enthalpy damping. In all cases three levels of FMG multigrid were used with 50 Runge-Kutta cycles on the coarser grids. Hence, all plots show the convergence for two sets of 50 cycles and then the convergence on the finest mesh. The plots are of the convergence rate of the residual of the continuity equation. For the original code this was updated for the density while in the preconditioned code it is updated for the pressure. Nevertheless, in the steady state the residual of the continuity equation should be the same except for the change in the artificial viscosity between the two algorithms. All cases were run with a matrix viscosity.

We first present two calculations for inviscid flow about a NACA 0012 . We use a $224 \times 32 \mathrm{C}$ mesh and three levels of multigrid. The first calculation is for inflow conditions $M=0.01, \alpha=1.25^{\circ}$. In this 
case we see that the residual asymptotes without the use of preconditioning and that the preconditioning dramatically increases the rate of convergence. The use of the preconditioning adds only a few percent to the total computational time. In the second case we consider the same geometry but with an inflow of $M=0.7, \alpha=1.25^{\circ}$. We have also done $M=0.8, \alpha=1.25^{\circ}$ which results in a minor slowing of the convergence rate. The preconditioned residual is the dotted line and the original code is the solid line. Different parameters for the time step and residual smoothing are needed with and without preconditioning. For inviscid cases we can choose $\beta_{\min }$ as zero while for the viscous cases $\beta_{\min }=0.4 \sqrt{u_{\infty}^{2}+v_{\infty}^{2}}$. For the transonic cases the lift and drag coefficients are changed only minimally by the preconditioning.

We next consider viscous flow about a RAE2822 airfoil on a $320 \times 64 \mathrm{C}$ mesh and 5 levels of multigrid on the finest grid with $M_{\infty}=0.01, \alpha=2.79^{\circ}$ using a Baldwin-Lomax turbulence model with Re $=6.5$ million. The residual history is presented in figure 3. Again the standard code converges very slowly for these low Mach numbers. In figure 4 we present both the preconditioned residual (dashed line) and the original code (solid line) for the same case but $M_{\infty}=0.73$. For viscous cases we choose $\beta_{\min }=0.4$. Again, for the transonic cases the lift and drag are changed by about 2 percent by the preconditioning. For the very low Mach numbers the lift and drag coefficients never converged for the non-preconditioned algorithm and seem to have significant errors. The preconditioned code gives much better agrees for lift and drag for low Mach numbers.

We conclude with a three dimensional case, inviscid flow about an ONERA wing. In figure 5 we display the convergence rate for the continuity equation (normalized by the initial residual) for Mach numbers .10 , .05 and .01 . We see that the convergence rate is independent of the inflow Mach number. In figure 6 we plot the lift coefficient for the same case. We again see that the lift coefficient is essentially independent of the Mach number except for some slight compressibility effects. Without preconditioning there are large variations in the lift for this set of Mach numbers. 


\section{$\underline{\text { Appendix }}$}

To find $\mathbf{P}_{p}$ we begin with the preconditioner $\mathbf{P}_{S}$ for the variables $d W_{S}=\left(\frac{d p}{\rho a}, d u, d v, d S\right)^{t}$, with $d S=$ $d p-a^{2} d \rho$. We then transform to $d W_{S}=(d p, d u, d v, d S)^{t}$ by multiplying all elements in the first row of the matrix by $\rho a$ and every element in the first column by $\frac{1}{\rho a}$. This gives

$$
\begin{aligned}
& \mathbf{P}_{\mathbf{S}}^{-1}=\left(\begin{array}{cccc}
\frac{a^{2}}{\beta^{2}} & 0 & 0 & 0 \\
\frac{\alpha a u}{\beta^{2}} & 1 & 0 & 0 \\
\frac{\alpha a v}{\beta^{2}} & 0 & 1 & 0 \\
0 & 0 & 0 & 1
\end{array}\right) \\
& \mathbf{P}_{\mathbf{S}}=\left(\begin{array}{cccc}
\frac{\beta^{2}}{a^{2}} & 0 & 0 & 0 \\
\frac{-\alpha u}{a} & 1 & 0 & 0 \\
\frac{-\alpha v}{a} & 0 & 1 & 0 \\
0 & 0 & 0 & 1
\end{array}\right)
\end{aligned}
$$

We then transform to the conservation variables $W_{c}=(\rho, \rho u, \rho v, E)^{t}$. This is given by $d W_{c}=T_{1} d W_{S}$. Let $\Gamma=\gamma-1$

$$
\begin{aligned}
& T_{1}=\left(\begin{array}{cccc}
\frac{1}{a^{2}} & 0 & 0 & -\frac{1}{a^{2}} \\
\frac{u}{a^{2}} & \rho & 0 & -\frac{u}{a^{2}} \\
\frac{v}{a^{2}} & 0 & \rho & -\frac{v}{a^{2}} \\
\frac{H}{a^{2}} & \rho u & \rho v & -\frac{M^{2}}{2}
\end{array}\right) \\
& T_{1}{ }^{-1}=\left(\begin{array}{cccc}
\Gamma G & -\Gamma u & -\Gamma v & \Gamma \\
-\frac{u}{\rho} & \frac{1}{\rho} & 0 & 0 \\
-\frac{v}{\rho} & 0 & \frac{1}{\rho} & 0 \\
\Gamma G-a^{2} & -\Gamma u & -\Gamma v & \Gamma
\end{array}\right)
\end{aligned}
$$

where $G=\frac{u^{2}+v^{2}}{2}$ and $a$ is the speed of sound. This gives the preconditioner in conservation variables.

Let $Q_{1}=\frac{(\gamma-1)\left(\beta^{2}-a^{2}\right)}{a^{4}}, Q_{2}=\frac{(\gamma-1)\left(\beta^{2}-(1+\alpha) a^{2}\right)}{a^{4}}, R=\frac{\beta^{2}}{a^{2}}-1+(\gamma-1) M^{2}\left(\frac{\left(\beta^{2}-a^{2}\right)}{2 a^{2}}-\alpha\right)$. Then in conservation variables $\mathbf{P}_{c}=T_{1} \mathbf{P}_{\mathbf{S}} \mathbf{T}_{\mathbf{1}}^{-\mathbf{1}}$,

$$
\mathbf{P}_{\mathbf{c}}=\left(\begin{array}{cccc}
1+G Q_{1} & -u Q_{1} & -v Q_{1} & Q_{1} \\
u G Q_{2} & 1-u^{2} Q_{2} & -u v Q_{2} & u Q_{2} \\
v G Q_{2} & -u v Q_{2} & 1-v^{2} Q_{2} & v Q_{2} \\
G R & -u R & -v R & 1+R
\end{array}\right)
$$

We next change from $w_{c}=(\rho, \rho u, \rho, E)$ variables to $w_{c}^{\prime}=\left(\rho, \rho u, \rho, E^{\prime}\right)$ variables, $E^{\prime}=E-\rho h_{\infty}+p_{\infty}$, $d W_{c}^{\prime}=T_{2} d W_{c}$.

$$
\begin{aligned}
T_{2} & =\left(\begin{array}{cccc}
1 & 0 & 0 & 1 \\
0 & 1 & 0 & 0 \\
0 & 0 & 1 & 0 \\
-h_{\infty} & 0 & 0 & 1
\end{array}\right) \\
\mathbf{P}_{\mathbf{c}}^{\prime} & =\left(\begin{array}{cccc}
1+G Q_{1} & -u Q_{1} & -v Q_{1} & -Q_{1} \\
u G Q_{2} & 1-u^{2} Q_{2} & -u v Q_{2} & u Q_{2} \\
v G Q_{2} & -u v Q_{2} & 1-v^{2} Q_{2} & v Q_{2} \\
R G & -u R & -v R & 1+R
\end{array}\right)
\end{aligned}
$$

Then $\mathbf{P}_{c}^{\prime}=\mathrm{T}_{2}\left(\mathrm{~T}_{1} \mathbf{P}_{\mathrm{S}} \mathrm{T}_{1}^{-1}\right) \mathbf{T}_{2}^{-1}$ 
We finally change only the time derivatives to the variables $W_{p}$ by $d W_{p}=T_{3} d W_{c}^{\prime}$.

$$
\begin{aligned}
T_{3} & =\left(\begin{array}{cccc}
\Gamma\left(G+h_{\infty}\right) & -\Gamma u & -\Gamma v & \Gamma \\
0 & 1 & 0 & 0 \\
0 & 0 & 1 & 0 \\
0 & 0 & 0 & 1
\end{array}\right) \\
T_{3}{ }^{-1} & =\left(\begin{array}{cccc}
\frac{1}{\Gamma\left(G+h_{\infty}\right)} & \frac{-u}{G+h_{\infty}} & \frac{-v}{G+h_{\infty}} & \frac{1}{G+h_{\infty}} \\
0 & 1 & 0 & 0 \\
0 & 0 & 1 & 0 \\
0 & 0 & 0 & 1
\end{array}\right)
\end{aligned}
$$

To summarize, we begin with

$$
\mathbf{P}_{S}^{-1} \frac{\partial W_{S}}{\partial t}+A_{S} \frac{\partial W_{S}}{\partial x}+B_{S} \frac{\partial W_{S}}{\partial y}=0
$$

and transform to

$$
\mathbf{P}_{c}^{-1} \frac{\partial W_{c}}{\partial t}+A_{c} \frac{\partial W_{c}}{\partial x}+B_{c} \frac{\partial W_{c}}{\partial y}=0
$$

We then transform, in conservation form, to the prime variables where $E$ is replaced by $E^{\prime}=E-h_{\infty} \rho$. Finally we then have that $\mathbf{P}_{p}^{-1}=\mathbf{P}_{c}^{\prime-1} T_{3}^{-1}=T_{2} T_{1} \mathbf{P}_{S}{ }^{-1} T_{1}^{-1} T_{2}^{-1} T_{3}^{-1}$. or $\mathbf{P}_{p}=T_{3} \mathbf{P}_{c}^{\prime-1}=T_{3} T_{2} T_{1} \mathbf{P}_{S} T_{1}^{-1} T_{2}^{-1}$. Thus,

$$
\mathbf{P}_{p}=\left(\begin{array}{cccc}
\Delta & -\frac{u \beta^{2}}{h} & -\frac{v \beta^{2}}{h} & \frac{\beta^{2}}{h} \\
-u \Delta B_{2} & 1+\frac{v^{2} \beta^{2} B_{2}}{h} & \frac{u v \beta^{2} B_{2}}{h} & \frac{-u \beta^{2} B_{2}}{h} \\
-v \Delta B_{2} & \frac{u v \beta^{2} B_{2}}{h} & 1+\frac{v^{2} \beta^{2} B_{2}}{h} & \frac{-v \beta^{2} B_{2}}{h} \\
-\Delta B_{4} & \frac{u \beta^{2} B_{4}}{h} & \frac{v \beta^{2} B_{4}}{h} & 1-\frac{\beta^{2} B_{4}}{h}
\end{array}\right)
$$

where all quantities were defined in the text.

We next show how to convert any preconditioner given in streamline coordinates and $\left(\frac{d p}{\rho a}, d u, d v, d S\right)$ coordinates to conservative variables in Cartesian (not streamwise) coordinates. We shall do this in two dimensions but the extension to three dimensions is straightforward. Assume we are given a preconditioner in streamline coordinates and $\left(\frac{d p}{\rho q}, d u, d v, d S\right)$ coordinates $P_{S}$ given by

$$
\mathbf{P}_{\mathbf{S}}=\left(\begin{array}{cccc}
P_{11} & P_{12} & 0 & 0 \\
P_{21} & P_{22} & 0 & 0 \\
0 & 0 & P_{33} & 0 \\
0 & 0 & 0 & P_{55}
\end{array}\right)
$$

We define rotation matrices $U, U^{-1}$ to get $P$ in Cartesian coordinates.

$$
\begin{aligned}
& U=\left(\begin{array}{cccc}
1 & 0 & 0 & 0 \\
0 & \cos \theta & \sin \theta & 0 \\
0 & -\sin \theta & \cos \theta & 0 \\
0 & 0 & 0 & 1
\end{array}\right) \\
& U^{-1}=\left(\begin{array}{cccc}
1 & 0 & 0 & 0 \\
0 & \cos \theta & -\sin \theta & 0 \\
0 & \sin \theta & \cos \theta & 0 \\
0 & 0 & 0 & 1
\end{array}\right)
\end{aligned}
$$

Let $q^{2}=u^{2}+v^{2}$. To get the streamwise direction we shall choose

$$
\cos \theta=\frac{u}{\sqrt{u^{2}+v^{2}}} \quad \sin \theta=\frac{v}{\sqrt{u^{2}+v^{2}}}
$$


Then the preconditioner in Cartesian coordinates is given by $\mathbf{P}_{\text {car }}=\mathbf{U}^{-1} \mathbf{P}_{\mathbf{S}} \mathbf{U}$ and

$$
\begin{aligned}
\mathbf{P}_{c a r} & =\left(\begin{array}{cccc}
P_{11} & P_{12} \frac{u}{q} & P_{12} \frac{v}{q} & 0 \\
P_{21} \frac{u}{q} & P_{22} \frac{u^{2}}{q^{2}}+P_{33} \frac{v^{2}}{q^{2}} & \left(P_{22}-P_{33}\right) \frac{u v}{q^{2}} & 0 \\
P_{21} \frac{v}{q} & \left(P_{22}-P_{33}\right) \frac{u v}{q^{2}} & P_{22} \frac{v^{2}}{q^{2}}+P_{33} \frac{u^{2}}{q^{2}} & 0 \\
0 & 0 & 0 & P_{55}
\end{array}\right) \\
& =\left(\begin{array}{cccc}
Q_{11} & Q_{12} & Q_{13} & 0 \\
Q_{21} & Q_{22} & Q_{23} & 0 \\
Q_{31} & Q_{32} & Q_{33} & 0 \\
0 & 0 & 0 & Q_{55}
\end{array}\right)
\end{aligned}
$$

We next introduce conservative variables $W_{c}$ as given in the appendix by the transformation $T_{1}$. The preconditioner for conservative variables is then given by $P_{c}=T_{1} P_{c a r} T_{1}^{-1}$

We now define the following quantities

$$
\begin{aligned}
Y_{1}= & u Q_{21}+v Q_{31}+w Q_{41} \\
Y_{2}= & u Q_{22}+v Q_{32}+w Q_{42} \\
Y_{3}= & u Q_{23}+v Q_{33}+w Q_{43} \\
Y_{4}= & u Q_{24}+v Q_{34}+w Q_{44} \\
Y_{5}= & (\gamma-1)\left(Q_{55}-Q_{11}\right) \\
G= & \frac{(\gamma-1) q^{2}}{2} \\
L= & (\gamma-1) \rho \\
Z_{11}= & \frac{1}{\rho a^{2}}\left[\frac{q^{2} Y_{5}}{2}-\frac{Y_{1}}{\rho}\right]+Q_{55} \\
Z_{12}= & \frac{1}{\rho a^{2}} u Y_{5}+\frac{Q_{12}}{\rho} \\
Z_{13}= & \frac{1}{\rho a^{2}} v Y_{5}+\frac{Q_{13}}{\rho} \\
Z_{14}= & \frac{1}{\rho a^{2}} w Y_{5}+\frac{Q_{14}}{\rho} \\
Z_{15}= & \frac{1}{\rho a^{2}} Y_{5} \\
Z_{51}= & H Z_{11}+G Y_{1}-u Y_{2}-v Y_{3}-w Y_{4} \\
& +Q_{55}\left(\frac{q^{2}}{2}-\frac{a^{2}}{\gamma-1}\right) \\
Z_{52}= & H Z_{12}-(\gamma-1) \rho u Y_{1}+Y_{2}-u Q_{55} \\
Z_{53}= & H Z_{13}-(\gamma-1) \rho v Y_{1}+Y_{3}-u Q_{55} \\
Z_{54}= & H Z_{14}-(\gamma-1) \rho w Y_{1}+Y_{4}-u Q_{55} \\
Z_{55}= & H Z_{15}+(\gamma-1) \rho Q_{55} \\
&
\end{aligned}
$$


Then

$$
\begin{gathered}
\mathbf{P}_{c}=\left(\begin{array}{cc}
Z_{11} & Z_{12} \\
u Z_{11}+G Q_{21}-Y_{2} & u Z_{12}-R u Q_{21}+Q_{22} \\
v Z_{11}+G Q_{31}-Y_{3} & v Z_{12}-R u Q_{31}+Q_{32} \\
w Z_{11}+G Q_{41}-Y_{4} & v Z_{12}-R u Q_{41}+Q_{42} \\
Z_{51} & Z_{52} \\
Z_{13} & Z_{14} \\
u Z_{13}-R v Q_{21}+Q_{23} & u Z_{14}-R w Q_{21}+Q_{24} \\
v Z_{13}-R v Q_{31}+Q_{33} & v Z_{14}-R w Q_{31}+Q_{34} \\
w Z_{13}-R v Q_{41}+Q_{43} & w Z_{14}-R w Q_{41}+Q_{44} \\
Z_{53} & Z_{54} \\
& \\
Z_{15} \\
v Z_{15}+R Q_{21} \\
v Z_{15}+R Q_{31} \\
w Z_{15}+R Q_{41} \\
Z_{55}
\end{array}\right)
\end{gathered}
$$

\section{$\underline{\text { References }}$}

1 Arnone A. and Turkel E., Pseudo-compressibilty Methods for the Incompressible Flow Equations, Proceedings 11th AIAA Computational Fluid Dynamics Conference, pp. 349-357, AIAA paper 93-3329, 1993

2 Chorin, A.J., A Numerical Method for Solving Incompressible Viscous Flow Problems, Journal of Computational Physics, 2 (1967), pp. 12-26.

${ }^{3}$ Godfrey, A.G., Walters, R.W. and Van Leer, B., Preconditioning for the Navier-Stokes Equations with Finite-Rate Chemistry, AIAA paper 93-0535, 1993.

${ }^{4}$ Gustafsson, B., and Stoor, H., Navier-Stokes Equations for Almost Incompressible Flow, SIAM J. Numer. Anal., 28(1991), pp. 1523-1547.

5 Jameson, A., Schmidt, W. and Turkel, E., Numerical Solutions of the Euler Equations by a Finite Volume Method using Runge-Kutta Time-Stepping Schemes, AIAA paper 81-1259, 1981.

${ }^{6}$ Lee, D. and Van Leer, B., Progress in Local Preconditioning of the Euler and Navier-Stokes Equations, Proceedings 11th AIAA CFD Conference, (1993) pp. 338-348.

7 Steger, J.L. and Kutler, P., Implicit Finite-Difference Procedures for the Computation of vortex wakes , AIAA J., 15(1977), pp. 581-590.

${ }^{8}$ Swanson, R.C., Turkel, E., Artificial Dissipation and Central Difference Schemes for the Euler and Navier-Stokes Equations, AIAA Computational Fluid Dynamics Conference, AIAA 87-1107-CP, 1987.

9 Swanson, R.C., Turkel, E., On Central Difference and Upwind Schemes , Journal of Computational Physics, 101(1992), pp. 292-306.

10 Turkel, E., Preconditioned Methods for Solving the Incompressible and Low Speed Compressible Equations, Journal of Computational Physics, 72(1987), 277-298.

11 Turkel, E., A Review of Preconditioning Methods for Fluid Dynamics, Applied Numerical Mathematics, 12(1993), pp. 257-284.

12 Van Leer, B., Lee, W.T., Roe, P.L., Characteristic Time-Stepping or Local Preconditioning of the Euler Equations, AIAA Paper 91-1552, 1991.

13 Volpe, G., Performance of Compressible Flow Codes at Low Mach Numbers, AIAA J., 31(1993), pp. 49-56. 


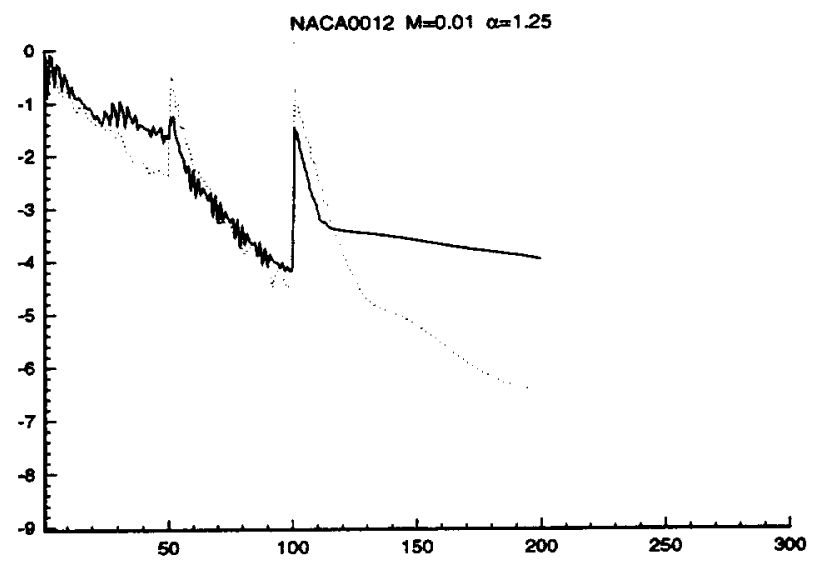

Figure 1: Convergence rate for inviscid flow about a NACA0012 with $M_{\infty}=0.01$ and $\alpha=1.25^{\circ}$

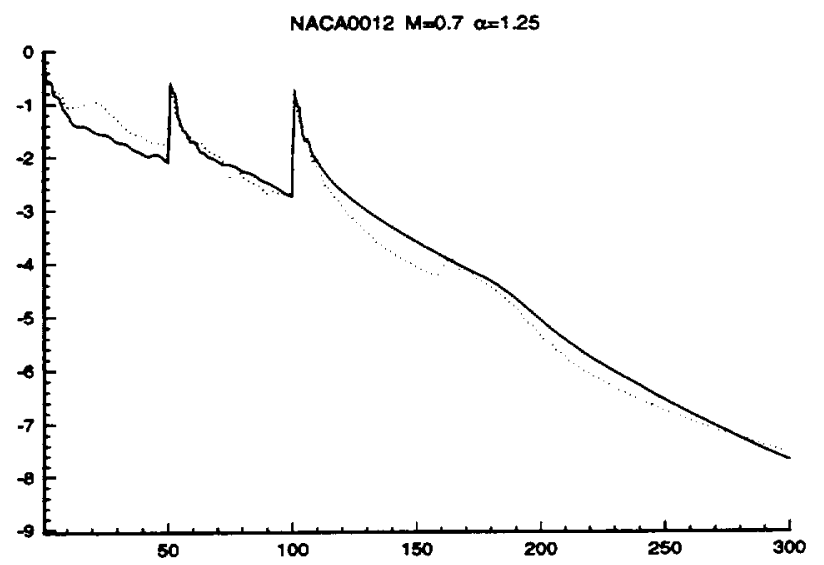

Figure 2: Same as figure 1 with $M_{\infty}=0.70$ and $\alpha=1.25^{\circ}$, dotted line is preconditioned scheme 


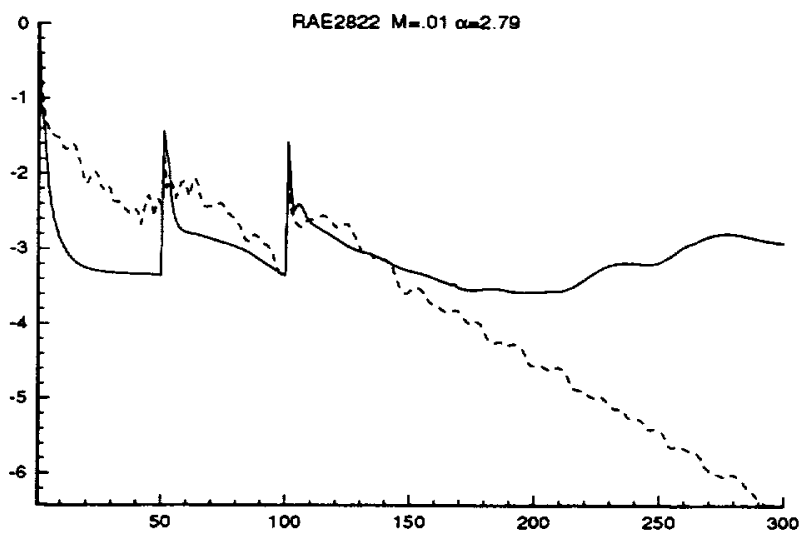

Figure 3: Convergence rate for viscous flow about a RAE2822 airfoil with $M_{\infty}=0.01$ and $\alpha=2.79^{\circ}$ Solid line is original algorithm and dashed line is the preconditioned scheme

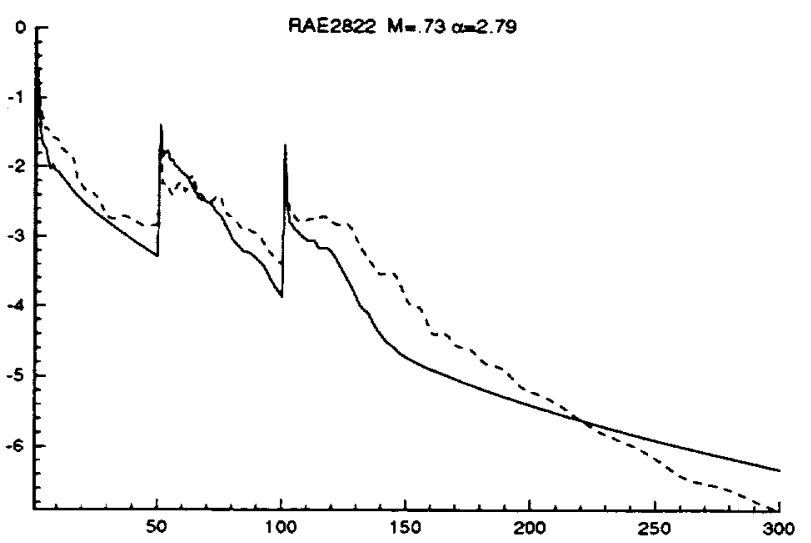

Figure 4: Convergence rate for viscous flow about a RAE2822 airfoil with $M_{\infty}=0.73$ and $\alpha=2.79^{\circ}$. Solid line is original algorithm and dashed line is the preconditioned scheme 


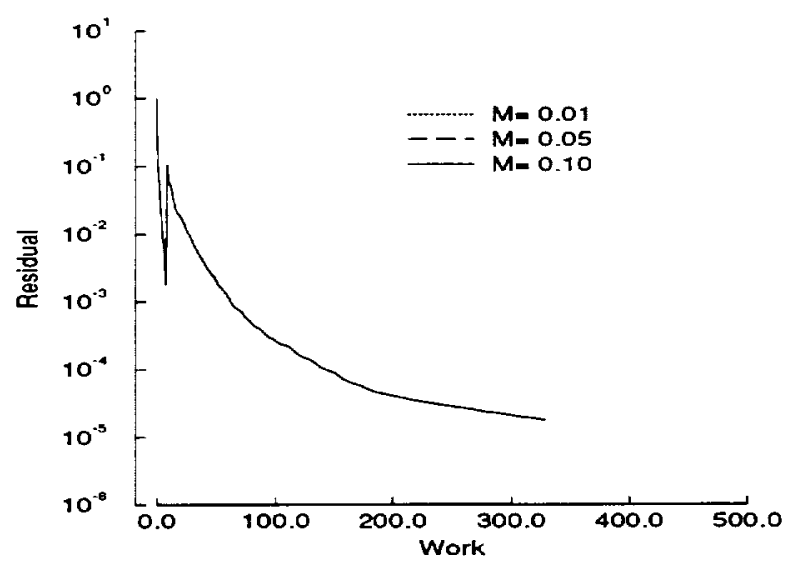

Figure 5: Convergence rate for inviscid flow about ONERA wing, $M_{\infty}=.10, .05, .01, \alpha=3.06^{\circ}$

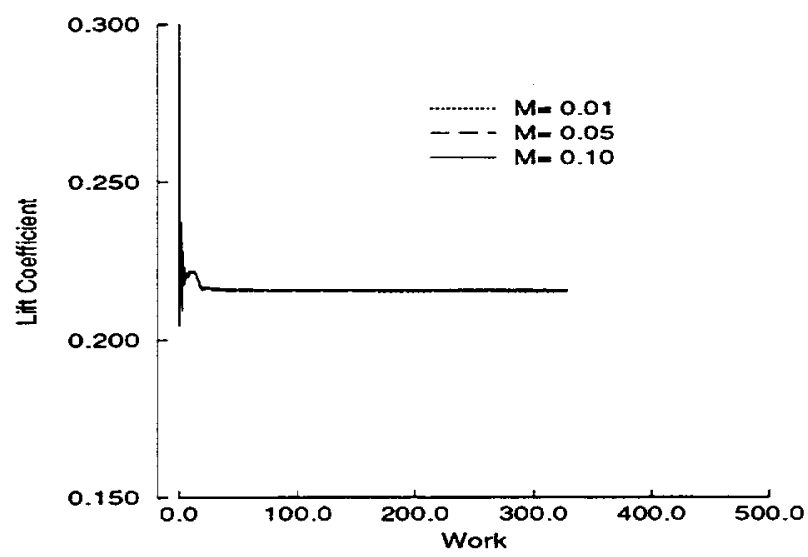

Figure 6: Lift coefficient for inviscid flow about a ONERA wing. 
Public reporting burden for this collection of information is estimated to average 1 hour per response. including the time for reviewing instructions, searching existing data sources, gathering and maintaining the data needed, and completing and reviewing the collection of information. Send comments regarding this burden estimate or any other aspect of this

Davis Highway. Suite 1204. Arlington, VA 22202-4302, and to the Office of Management and Budget, Paperwork Reduction Project (0704-0188), Washington, DC 20503.

1. AGENCY USE ONLY(Leave blank) $\begin{aligned} & \text { 2. REPORT DATE } \\ & \text { May } 1995\end{aligned}$
$\begin{aligned} & \text { 4. TITLE AND SUBTITLE } \\ & \text { PRESSURE UPDATING METHODS FOR THE STEADY-STATE } \\ & \text { FLUID EQUATIONS }\end{aligned}$
$\begin{aligned} & \text { 6. AUTHOR(S) } \\ & \text { Contractor Repor }\end{aligned}$
A. Fiterman
E. Turkel
V. Vatsa

7. PERFORMING ORGANIZATION NAME(S) AND ADDRESS(ES)

Institute for Computer Applications in Science and Engineering Mail Stop 132C, NASA Langley Research Center

8. PERFORMING ORGANIZATION REPORT NUMBER

ICASE Report No. 95-40 Hampton, VA 23681-0001

\section{SPONSORING/MONITORING AGENCY NAME(S) AND ADDRESS(ES)}

National Aeronautics and Space Administration

Langley Research Center

Hampton, VA 23681-0001

\section{FUNDING NUMBERS}

C NAS1-19480

WU 505-90-52-01

11. SUPPLEMENTARY NOTES

Langley Technical Monitor: Dennis M. Bushnell

Final Report

To appear in the Proc. of the AIAA CFD Conference

12a. DISTRIBUTION/AVAILABILITY STATEMENT

12b. DISTRIBUTION CODE

Unclassified-Unlimited

Subject Category 64

13. ABSTRACT (Maximum 200 words)

We consider the steady state equations for a compressible fluid. Since we wish to solve for a range of speeds we must consider the equations in conservation form. For transonic speeds these equations are of mixed type. Hence, the usual approach is to add time derivatives to the steady state equations and then march these equations in time. One then adds a time derivative of the density to the continuity equation, a derivative of the momentum to the momentum equation and a derivative of the total energy to the energy equation. This choice is dictated by the time consistent equations. However, since we are only interested in the steady state this is not necessary. Thus we shall consider the possibilty of adding a time derivative of the pressure to the continuity equation and similar modifications for the energy equation. This can then be generalized to adding combinations of time derivatives to each equation since these vanish in the steady state. When using acceleration techniques such as residual smoothing, multigrid, etc. these are applied to the pressure rather than the density. Hence, the code duplicates the behavior of the incompressible equations for low speeds.

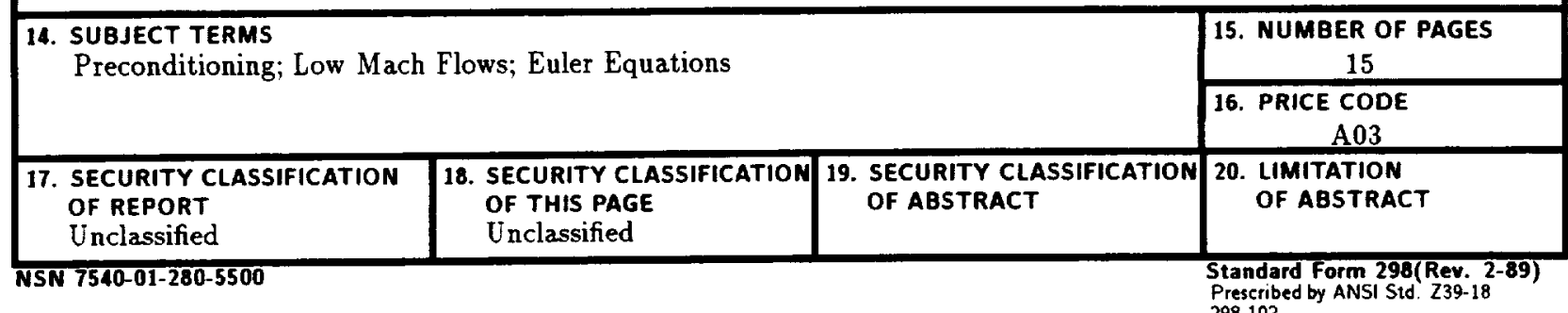

